Research Paper

\title{
The Immune Checkpoint Regulator PDL1 is an Independent Prognostic Biomarker for Biochemical Recurrence in Prostate Cancer Patients Following Adjuvant Hormonal Therapy
}

\author{
Heng Li1 ${ }^{1} 2^{*}$, Zhize Wang ${ }^{1,3^{*}}$, Yucong Zhang1,2, Guoliang Sun ${ }^{1,2}$, Beichen Ding1,2, Libin Yan ${ }^{1,2}$, Haoran Liu1,2, \\ Wei Guan ${ }^{1,2}$, Zhiquan $\mathrm{Hu}^{1,2}$, Shaogang Wang ${ }^{1,2}$, Fei Cheng ${ }^{5}$, Hua $\mathrm{Xu}^{1,2}{ }^{\bowtie}$, Xu Zhang4 ${ }^{4}$ Zhangqun Ye ${ }^{1,2}$ \\ 1. Hubei Institute of Urology, Tongji Hospital, Tongji Medical College, Huazhong University of Science and Technology, Wuhan 430030, China. \\ 2. Department of Urology, Tongji Hospital, Tongji Medical College, Huazhong University of Science and Technology, Wuhan 430030, China. \\ 3. Department of Urology, The First Affiliated Hospital, College of Medicine, Zhejiang University, Hangzhou 310003, China. \\ 4. Department of Urology/State Key Laboratory of Kidney Diseases, Chinese PLA General Hospital/PLA Medical School, Beijing 100000, China. \\ 5. Department of Pathology, The First Affiliated Hospital, School of Medicine, Zhejiang University, Hangzhou 310003, China. \\ *These authors contributed equally to this work.
}

$\square$ Corresponding author: Dr. Hua Xu, Department of Urology, Tongji Hospital, Tongii Medical College, Huazhong University of Science and Technology, Wuhan 430030, China. Hubei Institute of Urology, Tongji Hospital, Tongji Medical College, Huazhong University of Science and Technology, Wuhan 430030, China. Phone: 86-27-836-63454; Fax: 86-27-836-63454, Email: xuhuawhu@163.com

(c) Ivyspring International Publisher. This is an open access article distributed under the terms of the Creative Commons Attribution (CC BY-NC) license (https://creativecommons.org/licenses/by-nc/4.0/). See http://ivyspring.com/terms for full terms and conditions.

Received: 2018.10.03; Accepted: 2019.04.28; Published: 2019.06.02

\begin{abstract}
Background: The programmed death 1 (PDI)/programmed death ligand 1 (PDL1) targeted therapies have gained positive outcomes in several tumors, but the evidence of the expression and prognosis value of PDI/PDL1 in high risk prostate cancer was rare.

Methods: Immunohistochemical analysis of PDL1/PDI expression by a validated antibody was performed in a retrospectively collected high risk prostate cancer cohort who received adjuvant hormonal therapy (AHT) after radical prostatectomy (RP). The association between PDLI/PDI expression and prognosis was determined.

Results: In total, 127 patients were enrolled. $49.6 \%$ patients were considered PDL1-high expression while the PD1-positive expression proportion was $24.4 \%$. High PDL1 and negative PDI expression were significantly associated with lower prostate specific antigen (PSA) density $(p=0.010$ and $p=0.033$, respectively). Compared with the PDL1-low expression patients, the PDL1-high expression patients had significantly shorter time to PSA nadir (TTN) $(P=0.001)$ and biochemical recurrence $(B C R)(P=0.004)$. In Kaplan-Meier analysis, the PDL1-high expression group $(p<0.0001)$ and the PDL1-high/PDI-negative expression group $(p<0.0001)$ showed markedly lower BCR-free survival in localized disease. Univariate cause-specific Cox proportional hazard regression model concluded total PSA $(p=0.047)$, PDL1-high-expression $(p<0.001)$, PDL1-high/PD 1-negative expression $(p<0.001)$ were significant risk factors of shorter progression time to BCR in localized disease. PDLI-high-expression was the independent predictor of time to BCR in multiple Cox regression of all patients (Hazard ratio [HR]: 3.901; 95\% Confidence interval $[\mathrm{Cl}]$ : 1.287-11.824; $p=0.016$ ).

Conclusions: PDLl expression is not only highly prevalent in high-risk prostate cancer, but is also an independent biomarker in the prognosis of high-risk prostate cancer received AHT after RP. PDL1/PDI targeted therapy might be a potentially adjuvant treatment option for high-risk prostate cancer after RP.
\end{abstract}

Key words: PDL1, PD1, prostate cancer, adjuvant hormonal therapy, biomarker

\section{Introduction}

Prostate cancer is the second frequently diagnosed cancer in men for about $15 \%$ of all newly diagnosed male cancers worldwide. Prostate cancer is the fifth leading cause of death from cancer in men, 
with an estimated 307,000 deaths representing $6.6 \%$ of the total male cancer mortality ${ }^{1}$. First-line therapies for early stage localized prostate cancer include surgery and radiotherapy with approaches 100\% survival rate in 5-years 2, 3. For high-risk prostate cancer, AHT after radical prostatectomy significantly reduces the risk of disease progression in patients with localized or locally advanced prostate cancer 4,5 . Although AHT has been demonstrated to provide an initial benefit, but the majority of patients will progress BCR with adverse prognosis 6 .

PD1 is one of the immune checkpoint signaling which may induce $\mathrm{T}$ cell anergy and the differentiation of regulatory $\mathrm{T}$ cells whose functions contribute to further inhibition of antitumor immunity ${ }^{7}$. PD1 is mainly expressed on TReg cells to enhance their proliferation in the existence of a ligand 8 . Because many tumors are infiltrated with TReg cells, blockade of the PD1 pathway may also enhance antitumor immune responses by decreasing the number and/or repressing activity of intratumoral TReg cells9. One of the ligand of PD1 is PDL1, which may suggest the discrepancies antitumor activity of anti-PD1 antibody such as Nivolumab in castration-resistant prostate cancer (CRPC) ${ }^{10}$.

Recently studies about the expression level and the prognosis value of PD1/PDL1 in primary prostate cancer indicated that PDL1 is an independent indicator of $\mathrm{BCR}$ for radical prostatectomy ${ }^{11}$ while PD1 is a significant negative independent prognostic factor for clinical failure-free survival ${ }^{12}$. PD1 promoter methylation was also considered as a significant prognostic factor for BCR-free survival ${ }^{13}$, which could potentially raise the possibility of patients who might benefit from adjuvant PD1/PDL1 targeted treatment after radical prostatectomy.

Our study retrospectively enrolled 127 high risk prostate cancer patients who received AHT after RP in our hospital, the clinicopathological characteristics and prognostic value of PD1/PDL1 was assessed to give an early evidence of PD1/PDL1 targeted therapy might be a potential option for high risk prostate cancer after RP.

\section{Materials and Methods}

Patient characteristics and tissue samples. The included patients had histologically-confirmed prostate adenocarcinoma by needle biopsy, received radical prostatectomy, followed by adjuvant hormonal therapy including medical castration (luteinizing hormone releasing hormone analogue), combined with or without medical anti-androgen (bicalutamide, etc.) and had sufficient formalin-fixed paraffin-embedded matched archival tissue for immunohistochemistry. High-risk patients were included with a pT stage of $\geq 3$, surgical margin residual, $\mathrm{pN}$ stage $\geq 1$ or $\mathrm{pT}$ stage $\leq 2$ with high-risk factor (Gleason score $\geq 8$ or PSA $\geq 20 \mathrm{ng} / \mathrm{ml}$ ) $3,4,14$. Patients were excluded if they received additional concurrent anticancer therapies (chemotherapy or radiotherapy), nonstandard hormonal therapy or had other severe diseases. Demographic and clinical data for each patient were retrospectively collected from the hospital electronic patient record system.

We retrospectively collected 191 prostate cancer patients who were diagnosed with high risk prostate cancer received RP, followed by AHT at the Department of Urology, Tongji Hospital of Huazhong University of Science and Technology between the years 2010 and 2017. Among these patients, 64 were excluded because of additional concurrent anticancer therapies or nonstandard hormonal therapy. In total, 127 patients were enrolled in our study. The median time from diagnosis to data analysis was 40 months (IQR: 29-53 months).

Study design and assessments. This was a retrospectively study evaluating the ability of baseline (pretreatment) PDL1 status (high vs. low) and PD1 status (positive vs. negative) by immunohistochemistry to predict the risk of BCR following AHT in high risk prostate cancer received RP. This study was registered in the Chinese Clinical Trial Registry (NO ChiCTR-POC-17012719, http://www.chictr.org.cn/), and carried out in accordance with the ethical standards of the Helsinki Declaration and approved by the Tongji Hospital of Huazhong University of Science and Technology (Wuhan, China) ethics review committee (reference TJ-IRB20170801).

Follow-up assessments were retrospectively collected and included PSA measurements, prostate ultrasound scans, computed tomography (CT) of the chest, abdomen and pelvis, and technetium- $99 \mathrm{~m}$ bone scanning. Clinical investigators were unaware of the PDL1/PD1 status of the participants. All immunohistochemical slides were examined and scored by two experienced pathologists, who were blinded to all clinical data. If the staining intensity differed between the investigators, a third investigator evaluated the tissue sections, and the average score was recorded.

Informed consent statement. For experiments involving humans or human tissue samples, informed consent was obtained from all participants or their legal guardian/s.

Clinical outcomes. The primary events were biochemical recurrence, which was defined as an increase in the PSA level by $\geq 0.2 \mathrm{ng} / \mathrm{ml}$ for two consecutive measurements ${ }^{15}$, 16 in localized disease and was defined as an increase in the PSA level by 
$25 \%$ or more above the nadir (and by $\geq 2 \mathrm{ng} / \mathrm{ml}$ ), with confirmation four or more weeks later (PCWG3 criteria) $)^{17,18}$ in lymphatic or distant metastasis. Time to BCR was defined as the time from diagnosis to biochemical recurrence. The Time to PSA nadir (TTN) was defined as the duration of time from the initiation of treatment to the date the lowest PSA value. Survival status and the cause of death were recorded.

Immunohistochemistry and Evaluation. Immunohistochemistry was uniformly performed to assess protein expression of PDL1 (mouse monoclonal, ab210931, Abcam, Cambridge, UK, 1:200 dilution), PD1 (rabbit monoclonal, ab137132, Abcam, 1:200 dilution) by Bond Polymer Refine Detection System (Leica Biosystems Newcastle Ltd., Newcastle upon Tyne, UK). Omission of the primary antibody with phosphate-buffered saline served as a negative control for this detection system. We also performed immunohistochemical staining of human placenta tissues and tonsil tissues as positive controls for PD1 and PDL1 antibody respectively (Figure S1).

Since prostate cancer was considered heterogeneous, we selected regions where PDL1/PD1 expression was strongest to evaluate each tissue sample. Immunohistochemical staining intensity was evaluated independently by two pathologists who were blinded to patients' clinical outcome. The intensity of PDL1/PD1 positive cells was scored semi-quantitatively 11 as negative (0), weak (1), moderate (2), or strong (3). A semi-quantitative score was implemented to evaluate each tissue. The percentage of stained cells $(0-100 \%)$ was multiplied by the dominant intensity pattern of staining (0-3). Therefore, the overall semi-quantitative score ranged from 0 to 300. PDL1 expression was dichotomized by median, PD1 expression with staining intensity $\geq 2$ in more than $5 \%$ of tumour cells were considered as positive, similarly to previous studies ${ }^{19}$. A more detailed immunohistochemical protocol and the raw data of staining intensity score were given in the Supplement.

Antibody specificity. The specificity of the monoclonal anti-PDL1 antibodies was determined by western blotting using the prostate cancer cell lines LNCaP (Stem Cell Bank, Chinese Academy of Sciences, Shanghai, China) and 22Rv1 (Boster Biological Engineering Co. Ltd., Wuhan, China). Western blot was applied to testify the PDL1 protein expression, GAPDH was used as internal reference. Suppression of PDL1 protein was observed with siRNAs si1 (CGAATTACTGTGAAAGTCAAT) and si2 (GACCTATATGTGGTAGAGTAT), but not the negative control siRNA (Sigma-Aldrich, Munich, Germany).
Statistical Analysis. All statistical analyses were performed with IBM SPSS, v.21.0 (IBM, Armonk, NY) and GraphPad Prism 6 (La Jolla, CA). Divided PDL1 and PD1 expression by cut-off intensity score, associations between PDL1/PD1 expression and clinicopathological characteristics were analyzed. Continuous variables was presented as median (range) and compared by the Student's $t$ test; categorical data was presented as number (proportion) and compared by the chi-square test or Fisher's exact test. Pearson's correlation coefficient $r$ was appropriately calculated. The BCR-free survival was estimated by the Kaplan-Meier method and compared by log-rank test. Cox regression analysis was used to compare hazard ratios (HRs) in both univariate and multivariate models to evaluate the predictive role of all covariates for the risk of BCR. All statistical tests were two sided, $\mathrm{p}<0.05$ was considered significant.

\section{Results}

\section{PDLI antibody validation}

The anti-PDL1 antibody showed a relatively specific band around $33 \mathrm{KDa}$ in both LNCaP and 22Rv1 cell lines. To further demonstrate the ability of the antibody to detect PDL1, LNCaP and 22Rv1 cells were transfected with siRNAs targeting PDL1 or negative control. Note that siRNAs directly reduced PDL1 staining both in LNCaP and 22Rv1 cells, while control siRNA had no effect (Figure S2). Overall, these data indicate that the monoclonal antibody binds specifically to PDL1.

\section{Expression of PDLI and PDI in prostate cancer}

Among all enrolled patients, the proportion of PDL1-high expression was $49.6 \%(63 / 127)$ while the PD1-positive expression proportion was $24.4 \%$ (31/127). Representative immunohistochemical staining was shown in Figure 1. Not all malignant prostate cells expressed PDL1; PDL1 was expressed at the cytoplasm and membrane of tumor cells, occasionally detected in the nuclei. Stroma and tumor infiltrating lymphocytes also partially expressed PDL1. However, PD1 was mainly expressed at the membrane of stroma infiltrating lymphocytes.

\section{Patients' characteristics}

Clinicopathological characteristics of the patient included are shown in Table 1. High PDL1 and negative PD1 expression were found significantly associated with lower PSA density $(p=0.010$ and $p=0.033$, respectively). There is no statistically concordant between the expression of PDL1 and PD1 $(\mathrm{r}=0.449, \mathrm{p}=0.503)$. 


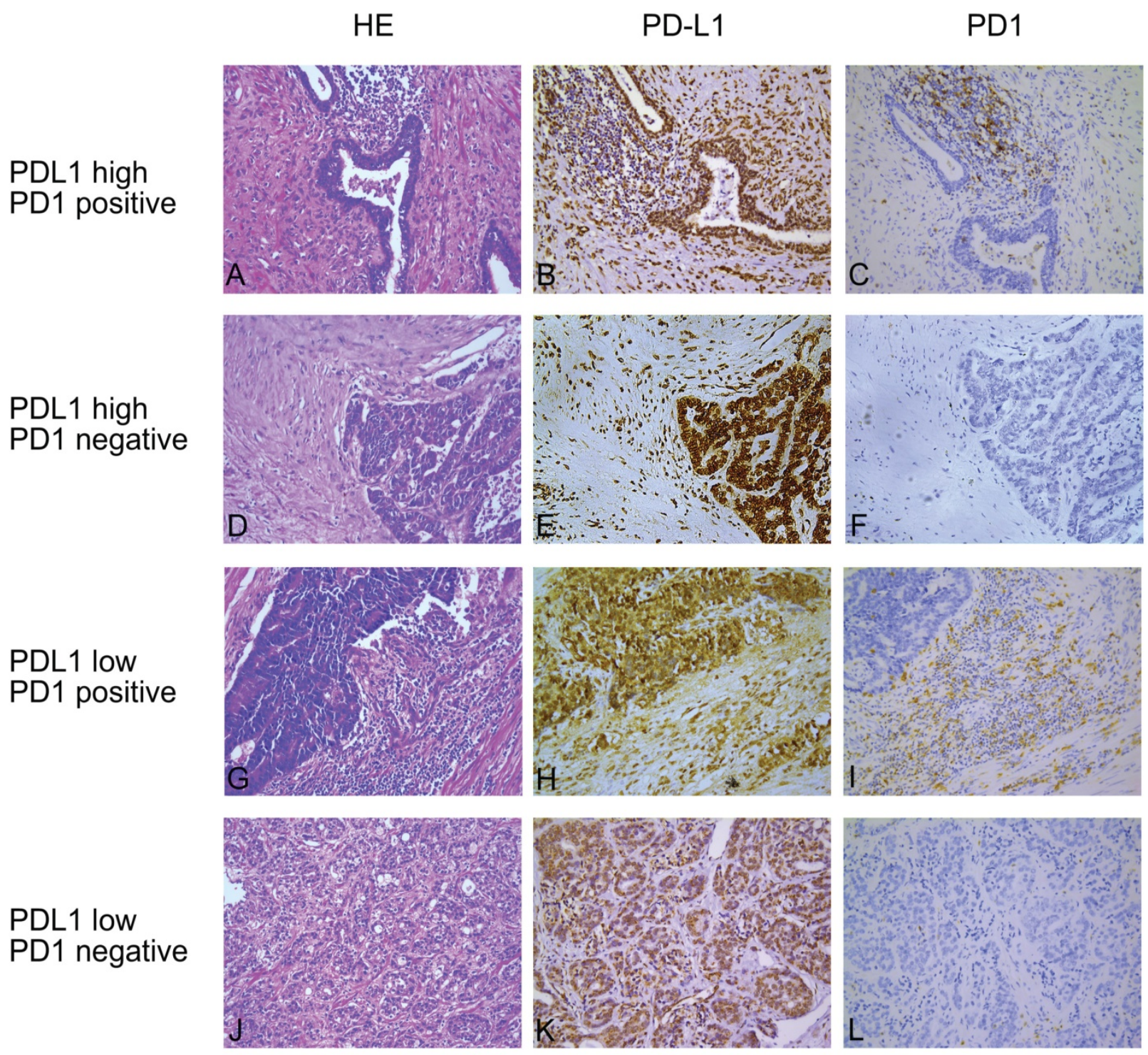

Figure 1. Representative hematoxylin-eosin (H\&E) and immunohistochemical (IHC) staining of PDLI and PDI. (A, D, G and J) are hematoxylin-eosin staining, (B, E, H and K) are immunohistochemical staining for programmed death ligand 1 (PDLI) and (C, F, I and L) are immunohistochemical staining for PDI in four consecutive tissue sections. The first and second rows are PDL1-high expression and the third and fourth rows are PDL1-low expression; the first and third rows are considered as PDI positive while the second and fourth rows are PDI negative. Original magnification: 200x.

Table 1. Baseline clinicopathological characteristics of all enrolled patients.

\begin{tabular}{|c|c|c|c|c|c|c|c|}
\hline \multirow[t]{2}{*}{ Characteristics } & \multicolumn{7}{|l|}{ Results } \\
\hline & Total & PD-L1 high & PD-L1 low & p-value ${ }^{a}$ & PD-1 positive & PD-1 negative & p-value ${ }^{a}$ \\
\hline No. of patients (\%) & 127 & $63(49.6)$ & $64(50.4)$ & & $31(24.4)$ & $96(75.6)$ & \\
\hline Age (median, range) & $66(48-76)$ & $66.5(48-76)$ & 66(53-75) & 0.581 & $67.5(48-76)$ & $65.5(48-75)$ & 0.134 \\
\hline \multicolumn{8}{|l|}{ Gleason score (n, \%) } \\
\hline$\leq 7$ & $64(49.6)$ & $28(43.8)$ & $36(56.3)$ & & $12(18.8)$ & $52(81.3)$ & \\
\hline$\geq 8$ & $63(50.4)$ & $35(55.6)$ & $28(44.4)$ & 0.124 & 19(30.2) & $44(69.8)$ & 0.098 \\
\hline \multicolumn{8}{|c|}{ Pathological T stage $(\mathrm{n}, \%)$} \\
\hline $\mathrm{pT}_{2}$ & $54(42.5)$ & $26(48.1)$ & $28(51.9)$ & & $14(25.9)$ & $40(74.1)$ & \\
\hline $\mathrm{pT}_{3} / \mathrm{pT}_{4}$ & $73(57.5)$ & $37(50.7)$ & $36(49.3)$ & 0.459 & $17(23.3)$ & $56(76.7)$ & 0.445 \\
\hline \multicolumn{8}{|c|}{ Pathological N stage (n, \%) } \\
\hline $\mathrm{pN}_{0}$ & $86(67.7)$ & $44(51.2)$ & $42(48.8)$ & & 18(20.9) & 68 (79.1) & \\
\hline$\geq \mathrm{pN}_{1}$ & $41(32.3)$ & 19(46.3) & $22(53.7)$ & 0.375 & $13(31.7)$ & $28(68.7)$ & 0.136 \\
\hline
\end{tabular}




\begin{tabular}{|c|c|c|c|c|c|c|c|}
\hline \multirow[t]{2}{*}{ Characteristics } & \multicolumn{7}{|l|}{ Results } \\
\hline & Total & PD-L1 high & PD-L1 low & p-value ${ }^{a}$ & PD-1 positive & PD-1 negative & p-value \\
\hline R0 & $80(63.0)$ & $38(47.5)$ & $42(52.5)$ & & $16(20.0)$ & $64(80.0)$ & \\
\hline R1 & $47(37.0)$ & 25(53.2) & $22(46.8)$ & 0.332 & $15(31.9)$ & $32(68.1)$ & 0.098 \\
\hline Preoperative Total prostate-specific antigen(mean, range, $\mathrm{ng} / \mathrm{ml}$ ) & $\begin{array}{l}49.74 \\
(1.98-408.21)\end{array}$ & $\begin{array}{l}40.36 \\
(1.98-408.21)\end{array}$ & $\begin{array}{l}59.14 \\
(6.61-307.59)\end{array}$ & 0.131 & $\begin{array}{l}40.09 \\
(2.78-307.59)\end{array}$ & $\begin{array}{l}52.83 \\
(1.98-408.21)\end{array}$ & 0.382 \\
\hline Prostate volume (mean, range, $\mathrm{cm}^{3}$ ) & $\begin{array}{l}72.61 \\
(33.79-180.03)\end{array}$ & $\begin{array}{l}74.85 \\
(33.38-180.03)\end{array}$ & $\begin{array}{l}70.06 \\
(33.79-132.32)\end{array}$ & 0.455 & $\begin{array}{l}61.75 \\
(33.79-95.97)\end{array}$ & $\begin{array}{l}74.96 \\
(34.17-180.03)\end{array}$ & 0.113 \\
\hline Prostate-specific antigen density (mean, range, $\mathrm{ng} / \mathrm{ml} / \mathrm{cm}^{3}$ ) & $\begin{array}{l}0.89 \\
(0.03-9.10)\end{array}$ & $\begin{array}{l}0.522 \\
(0.03-2.27)\end{array}$ & $\begin{array}{l}1.31 \\
(0.11-9.09)\end{array}$ & 0.010 & $\begin{array}{l}1.623 \\
(0.03-9.09)\end{array}$ & $\begin{array}{l}0.745 \\
(0.03-3.12)\end{array}$ & 0.033 \\
\hline PD-L1 high (n, \%) & 63 & - & - & & $17(27.0)$ & $46(73.0)$ & 0.332 \\
\hline PD-L1 low (n, \%) & 64 & - & - & & $14(21.9)$ & $50(78.1)$ & \\
\hline PD-1 positive (n, \%) & 31 & $17(54.8)$ & $14(45.2)$ & & - & - & \\
\hline PD-1 negative (n, \%) & 96 & $46(47.9)$ & $50(52.1)$ & & - & - & \\
\hline
\end{tabular}

$a, p$ values are based on Fisher's exact test and Student's $t$ test for categorical and continuous variables, respectively.

A

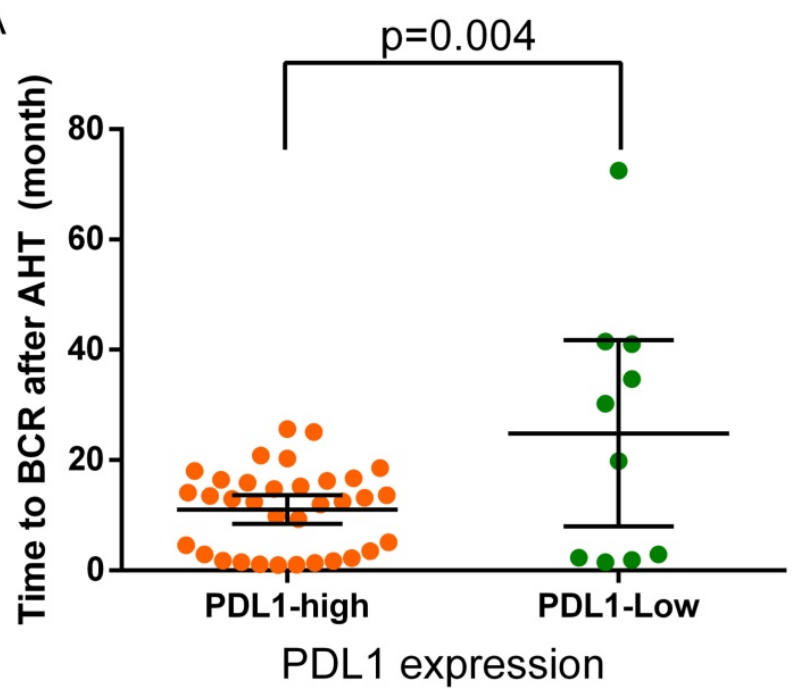

B

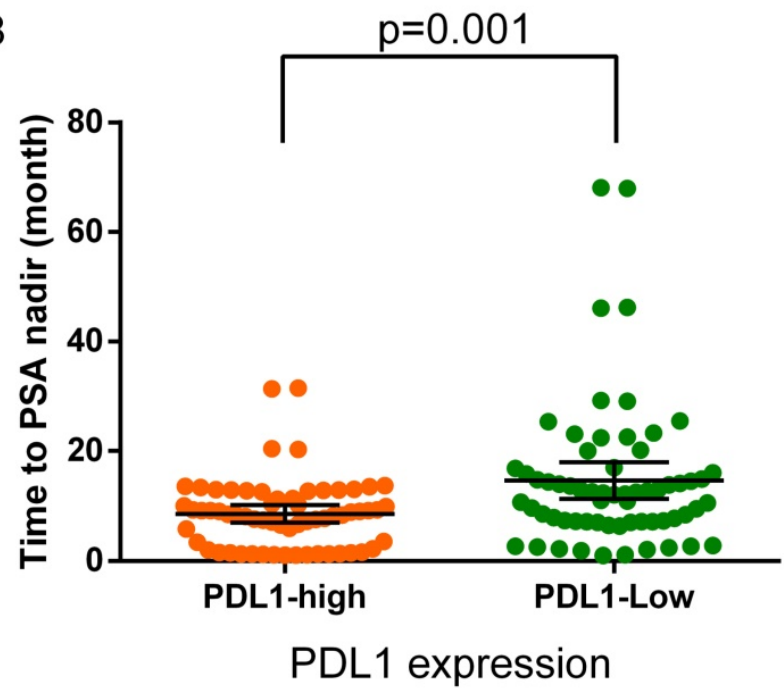

Figure 2. (A) The time to biochemistry recurrence (BCR) after adjuvant hormonal therapy in the PDL1-high and PDL1-low expression groups. Black crosshairs denote the mean time to BCR with $95 \% \mathrm{Cl}$. (B) The time to PSA nadir (TTN) after adjuvant hormonal therapy in the PDL1-high and PDL1-low expression groups. Black crosshairs denote the mean TTN with $95 \% \mathrm{Cl}$. p values are for Student's $t$ tests.

\section{High PDLI expression related to the worse prognosis of AHT}

During the follow-up, the overall proportion of patients progressed to biochemistry recurrence during AHT was $34.6 \%(44 / 127)$ with a median time of 13 months (range: 1-73 months). The proportion of BCR among PDL1-high-expression patients was $54.0 \%$ (34 of 63 ), whereas $15.6 \%$ (10 of 64) in PDL1-low-expression patients. The median time to BCR in PDL1-high-expression patients was 13 months (range: 1-26 months), whereas 25 months (range: 1.5-73 months, $\mathrm{p}=0.004$ ) in PDL1-low-expression patients (Figure 2a). The median time to PSA nadir after adjuvant hormonal therapy was 9 months (range: 1-68 months). In PDL1-high-expression patients the median TTN was 8 months (range: 1-32 months) while 12 months (range: 1-68 months, $\mathrm{p}=0.001$ ) in PDL1-low-expression patients (Figure 2b).

\section{PDL1/PDI status is a risk factor of BCR after AHT}

The median BCR-free survival in PDL1-high-expression patients was 18.5 months (95\% CI: 13.256-23.924), while in PDL1-low-expression patients it was 72.5 months (95\% CI: 29.106-115.954). We further stratified patients into localized and metastatic disease. In localized disease, Kaplan-Meier analyses indicated that BCR-free survival was dramatically lower in high-PDL1-expression patients with median BCR-free survival 16 months (95\% CI: 14.006-18.514) than low-PDL1-expression patients 72.5 months (95\% CI: 28.982-116.078; $\mathrm{p}<0.0001$ ) but not associated with PD1 status. The BCR-free survival was further analyzed according to the panel of PDL1/PD1 status of localized disease and statistical significance was concluded $(p<0.0001)$. The median BCR-free survival in PDL1-high/PD1-negative expression patients was 16 months $(95 \%$ CI: 13.527-18.213), while PDL1-high/PD1-positive or PDL1-low/PD1-negative expression patients was 72.5 
months (95\% CI: 28.957-116.103). In metastatic disease, BCR-free survival was not significantly associated with PDL1/PD1 status. Of note, no PDL1-low/PD1-positive expression patients occurred $\mathrm{BCR}$ in our surveillance (Figure 3 ).

Further analysis of BCR free survival stratified by different PDL1/PD1 status was shown in Figure 4. High-PDL1 expression was significantly associated with lower BCR-free survival both in PD1-positive $(p=0.0193)$ and PD1-negative patients $(p<0.0001)$.

\section{PDLI is an independent prognostic biomarker of BCR after AHT}

In univariate cause-specific Cox proportional hazard regression model of localized disease, total

A

Biochemical recurrence free survival of localized disease

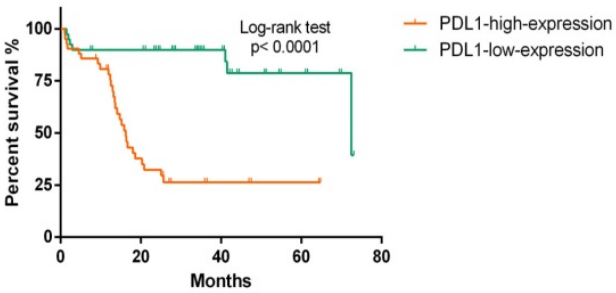

No. at Risk

$\begin{array}{lrrrrr}\text { PDL1-high expression } & 42 & 15 & 5 & 3 & 0 \\ \text { PDL1-low expression } & 40 & 35 & 19 & 7 & 0\end{array}$

B

Biochemical recurrence free survival of localized disease

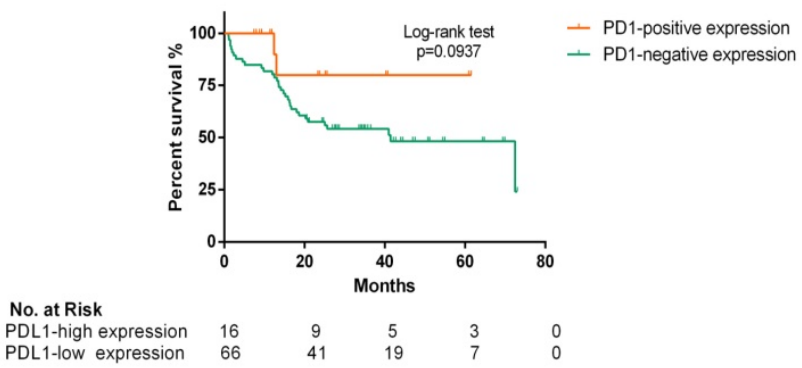

No. at Risk

$\begin{array}{llcccc}\text { PDL1-high expression } & 16 & 9 & 5 & 3 & 0 \\ \text { PDL1-low expression } & 66 & 41 & 19 & 7 & 0\end{array}$

C

Biochemical recurrence free survival of localized disease

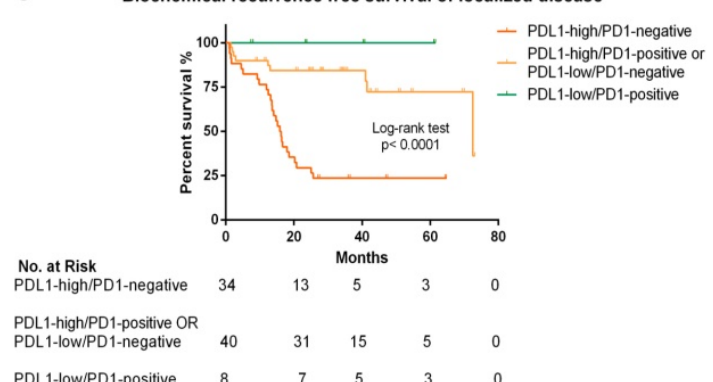

prostate specific antigen (HR: 2.077; 95\% CI: 1.010-4.270; $\mathrm{p}=0.047)$, PDL1-high expression (HR: 7.295; 95\%CI: 2.981-17.852; p<0.001), PDL1-high/ PD1-negative expression (HR: 6.330; 95\%CI: 2.843-14.095; $\mathrm{p}<0.001$ ) were significant risk factors of shorter progression time to BCR. In multivariate Cox proportional hazards model analysis of all patients, PDL1-high expression (HR: 3.901; 95\%CI: 1.287-11.824; $p=0.016$ ) remains an independent predictor of time to $\mathrm{BCR}$ after adjusting for time to PSA nadir, PDL1/PD1 status and N stage at diagnosis (Table 2).

D

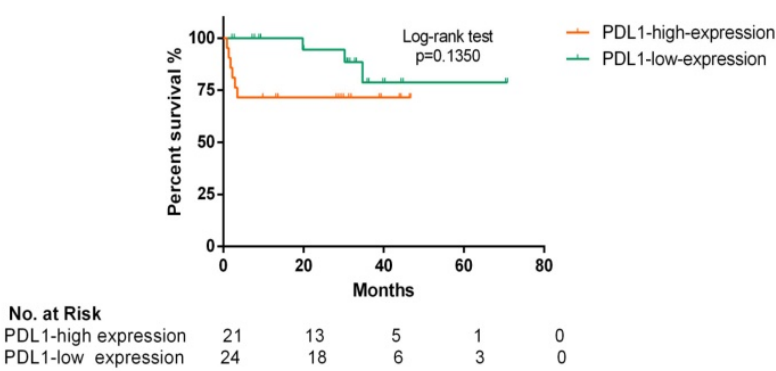

Biochemical recurrence free survival of metastatic disease

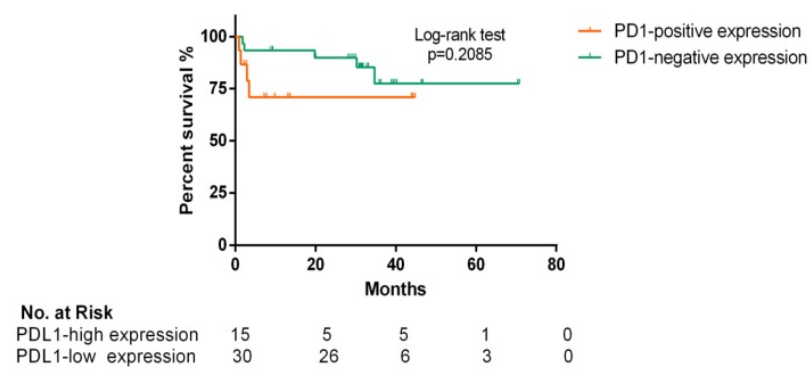

$\mathbf{F}$ Biochemical recurrence free survival of metastatic disease

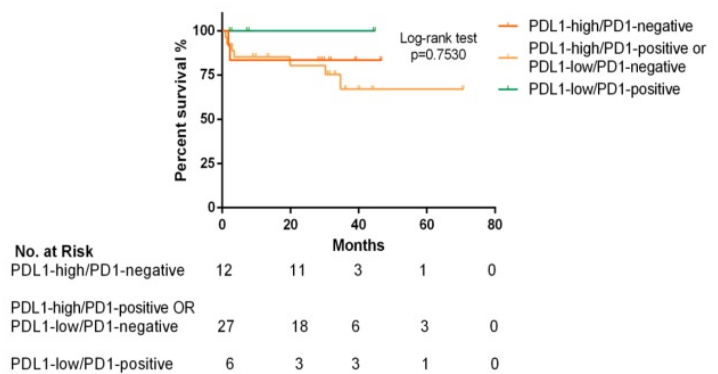

Figure 3. Kaplan-Meier analysis of biochemical recurrence free survival according to PDL1/PD1 status in patients stratified by localized or metastatic disease (A, B, C, D, E and F). (A) The hazard ratio for BCR progression with PDL1-high-expression in localized disease was 6.087 ( $95 \%$ Cl: 3.028-11.73; $\mathrm{P}<0.0001$ by the log-rank test). (B) The hazard ratio for BCR progression with PDI positivity in localized disease was 0.317 ( $95 \% \mathrm{Cl}: 0.186-1.134$; $\mathrm{P}=0.094$ by the log-rank test). (C) The hazard ratio for $\mathrm{BCR}$ progression with the panel of PDL1-high/PDI-negative status expression in localized disease was 6.330 (95\% Cl: $2.843-14.095 ; p<0.0001$ by the log-rank test) (D) .The hazard ratio for BCR progression with PDL1-high-expression in metastatic disease was 2.746 ( $95 \% \mathrm{Cl}: 0.733-10.33$; $\mathrm{p}=0.135$ by the log-rank test). (E) The hazard ratio for BCR progression with PD1 positivity in metastatic disease was $2.253(95 \% \mathrm{Cl}: 0.593-11.580 ; \mathrm{p}=0.2085$ by the log-rank test). $(\mathrm{F})$ The hazard ratio for $\mathrm{BCR}$ progression with the panel of PDL1-high/PDI-negative status expression in metastatic disease was $0.761(95 \% \mathrm{Cl}: 0.158-3.668 ; \mathrm{p}=0.753$ by the log-rank test) 
$A$

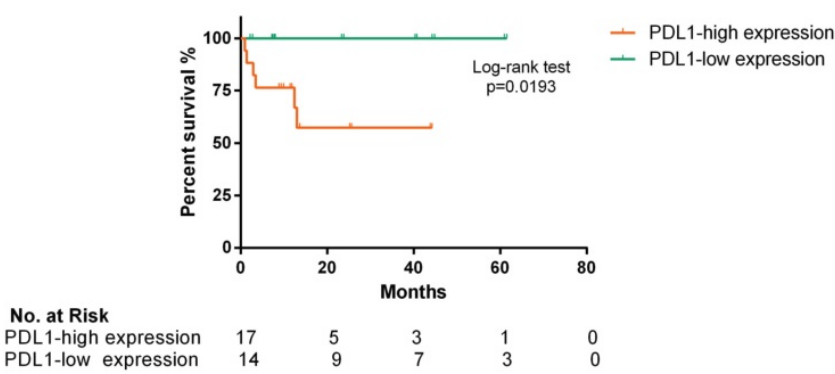

C

BCR free survival of PDL1-high expression patients

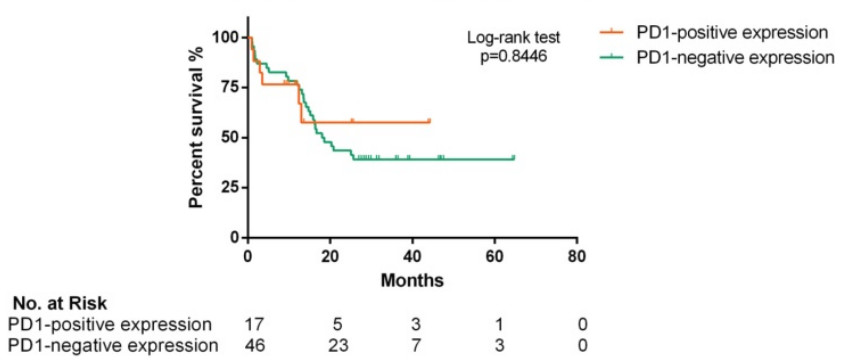

B

BCR free survival of PD1-negative patients

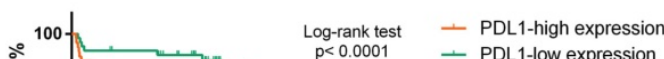

Log-rank test
$\mathrm{p}<0.0001$

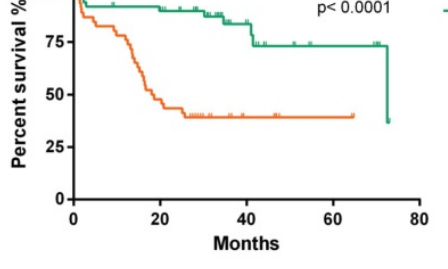

No. at Risk

PDL1-high expression

$\begin{array}{rrrrr}46 & 23 & 7 & 3 & 0 \\ 50 & 44 & 18 & 7 & 0\end{array}$

BCR free survival of PDL1-low expression patients

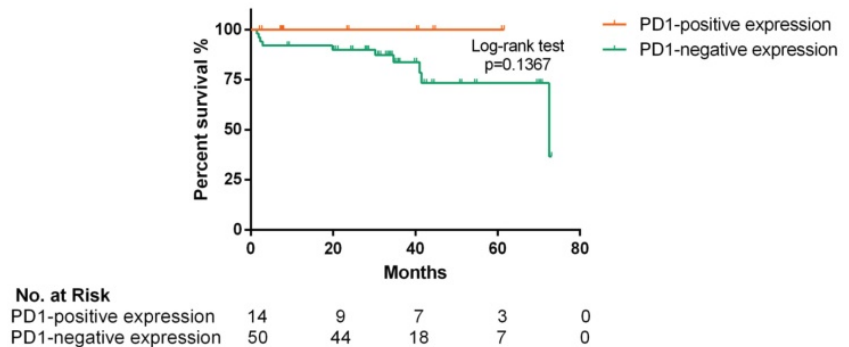

Figure 4. Kaplan-Meier analysis of biochemical recurrence free survival in all enrolled patients according to different PDL1/PD1 status (A, B, C and D). (A) The hazard ratio for BCR progression with PDL1-high expression in PDI-positive patients was 6.799 ( $95 \% \mathrm{Cl}: 1.365-33.85$; $\mathrm{P}=0.0193$ by the log-rank test). (B) The hazard ratio for $\mathrm{BCR}$ progression with PDL1-high expression in PD1-negative patients was 4.344 ( $95 \% \mathrm{Cl}: 2.333-8.583 ; \mathrm{p}<0.0001$ by the log-rank test). (C) The hazard ratio for BCR progression with PD 1 -positive expression in PDL1-high expression patients was 0.917 ( $95 \% \mathrm{Cl}: 0.389-2.162 ; \mathrm{p}=0.8446$ by the log-rank test). (D) The hazard ratio for BCR progression with PDI-positive expression in PDLI-low expression patients was 0.2865 ( $95 \% \mathrm{Cl}: 0.055-1.486 ; \mathrm{p}=0.1367$ by the log-rank test)

Table 2. Univariate and multivariate Cox analyses of BCR-free survival in patients received AHT after radical prostatectomy.

\begin{tabular}{|c|c|c|c|c|c|c|}
\hline \multirow[t]{3}{*}{ Variable } & \multicolumn{4}{|l|}{ Univariate analysis } & \multicolumn{2}{|l|}{ Multivariate analysis } \\
\hline & \multicolumn{2}{|l|}{ Localized disease } & \multicolumn{2}{|l|}{ Metastatic disease } & \multicolumn{2}{|l|}{ All patients } \\
\hline & HR $(95 \% \mathrm{CI})$ & p-value & $\mathrm{HR}(95 \% \mathrm{CI})$ & p-value ${ }^{a}$ & $\operatorname{HR}(95 \% \mathrm{CI})$ & p-value \\
\hline Age at diagnosis(ref: $\leq 66.0$ years) & $0.868(0.442-1.707)$ & 0.682 & $1.693(0.438-6.548)$ & 0.445 & - & - \\
\hline \multicolumn{7}{|l|}{$\begin{array}{l}\text { Gleason score at diagnosis } \\
\text { (ref: } 6 \leq G \leq 7 \text { ) }\end{array}$} \\
\hline Gleason score $\geq 8$ & $0.915(0.458-1.830)$ & 0.802 & $0.250(0.031-2.011)$ & 0.193 & - & - \\
\hline \multicolumn{7}{|l|}{$\mathrm{T}$ stage at diagnosis } \\
\hline $\mathrm{T}_{3} / \mathrm{T}_{4}\left(\mathrm{ref}: \leq \mathrm{T}_{2}\right)$ & $0.693(0.352-1.364)$ & 0.289 & $3.711(0.996-13.830)$ & 0.051 & - & - \\
\hline \multicolumn{7}{|l|}{$\mathrm{N}$ stage at diagnosis } \\
\hline $\mathrm{N}_{1}\left(\right.$ ref: $\left.\mathrm{N}_{0}\right)$ & - & - & - & - & $0.355(0.157-0.807)$ & 0.013 \\
\hline Total prostate-specific antigen $(\mathrm{ng} / \mathrm{ml})(\mathrm{ref}: \leq 29.76)$ & $2.077(1.010-4.270)$ & 0.047 & $0.591(0.147-2.384)$ & 0.460 & - & - \\
\hline $\begin{array}{l}\text { Surgical Margin status } \\
\text { (ref: negative) }\end{array}$ & $0.903(0.425-1.918)$ & 0.791 & $3.502(0.721-17.007)$ & 0.120 & - & - \\
\hline Time to PSA nadir (ref: $<9.2$ month) & $1.732(0.874-3.435)$ & 0.116 & $3.569(0.874-14.574)$ & 0.076 & $1.181(0.857-1.628)$ & 0.310 \\
\hline PDL1 status(ref: low) & $7.295(2.981-17.852)$ & $<0.001$ & $2.763(0.688-11.094)$ & 0.152 & $3.901(1.287-11.824)$ & 0.016 \\
\hline PD1 status(ref: negative) & $0.314(0.075-1.313)$ & 0.113 & $2.303(0.606-8.761)$ & 0.221 & - & - \\
\hline $\begin{array}{l}\text { PDL1 high/PD1 negative (ref: PDL1 low/PD1 positive OR PDL1 high/PD1 positive } \\
\text { OR PDL1 low/PD1 negative) }\end{array}$ & $6.330(2.843-14.095)$ & $<0.001$ & $0.761(0.158-3.668)$ & 0.733 & $1.109(0.445-2.759)$ & 0.825 \\
\hline
\end{tabular}

$\mathrm{a}$, Individual covariates were tested to predict outcome using a univariate Cox proportional hazards model. $\mathrm{b}$, $\mathrm{p}$ values are the result of a adjusted multivariable Cox proportional hazards model.

\section{Discussion}

Since the dependence of prostate cancer on androgen signaling firstly discovered by Huggins and Hodges ${ }^{20}$, hormonal therapy has been considered as the standard treatment for metastatic and locally advanced disease. Adjuvant hormonal therapy aimed to treat the residual tumor in the surgical margin, pathological positive lymphocyte, micro-transplant lesion after radical prostatectomy or radical radiotherapy in order to improve long-term survival21, 22. Early AHT has shown significantly cancer-specific survival and overall survival improvement in patients who proved to be high-risk after RP in a prospective randomized trial ${ }^{6}$, while there was no statistically prognostic difference in patients with minimal nodal from retrospective studies $^{23}, 24$. The findings suggested multimodal strategies for high-risk prostate cancer, of which RP was an important component. Nevertheless, these 
patients would experience BCR and have adverse prognosis ${ }^{25}$, which is consist with our survival surveillance data: the median BCR-free survival for patients received AHT after $\mathrm{RP}$ is 13 months.

Recently, several new biomarkers were discovered the value of treatment response prediction, such as AR-V7 ${ }^{26}$. New focus on the interaction between host immune response and cancer is particularly on PD1 and PDL1 which were regarded as major immune modulators in various tumor entities ${ }^{27}$. Meanwhile, PD1 checkpoint inhibitors have gained regulatory approval for the treatment of several metastasized malignant cancer 28 , 29. In prostate cancer, the utility of anti-PDL1/PD1 pathway therapy is still under consideration ${ }^{10}$, varies ongoing clinical trials are investigating the use of PD1/PDL1 agents in prostate cancer (NCT02312557, NCT02458638).

Depending on the antibody used, the reported rates of PDL1 positivity in primary prostate cancer ranged greatly. Several studies have been adequately validated the robust sensitivity and specificity of staining protocols and PDL1 antibodies in large cohorts of primary prostate cancers and reported a positivity rate between $8-20 \% 30-32$. Gevensleben et al have shown that the PD1 receptor ligand PDL1 is differentially expressed among primary prostate cancer patients and validated a PDL1 monoclonal antibody EPR116111. The moderate to high expression of PDL1 in primary prostate cancer after radical prostatectomy is $52.2-61.7 \%$. Consistently, we used this cut-off value to assess the positive rate of PDL1 in the high-risk prostate cancer patients received AHT after RP in our cohort was $49.6 \%$. Line with our previous research 33 , the PDL1/PD1 status was assessed by a specific and feasible immunohistochemistry method. A validated PDL1 monoclonal antibody, together with a PD1 monoclonal antibody was used to evaluate the PDL1/PD1 status in the FFPE tissue of enrolled patients. Likely with the Ness et al ${ }^{12}$, PDL1 commonly express in malignant epithelial cells and stroma cells of primary prostate cancer, while PD1 was mainly seen in lymphocytes in prostate cancer interstitial substance.

Through analyzing the methylation of PD1 promoter, Goltz et al demonstrated PD1 methylation plays a significant prognostic role for biochemical recurrence (BCR)-free survival. PD1 expression in prostate cancer should be identified for patients who intended to receive adjuvant treatment after radical prostatectomy ${ }^{13}$. Gevensleben et al 34 determined PDL1 promoter methylation in 797 patients undergoing radical prostatectomy and found it was associated with BCR. These findings raised a common concern about the prognostic value of mPDL1 and mPD1 in the patients after RP. Encouraged by these studies, we evaluate the PDL1/PD1 status in high risk prostate cancer received AHT after RP by immunohistochemistry, the survival surveillance showed the median BCR-free survival in PDL1-high expression patients was 18.5 months, while in PDL1-low expression patients was 72.5 months. The Cox regression shows the hazard ratio for $\mathrm{BCR}$ progression in localized disease with high PDL1 expression is 7.295 (95\% CI: (2.981-17.852; p<0.001). In line with the two large independent cohorts enrolled in Gevensleben's study ${ }^{11}$, high-PDL1 expression was significantly associated with worse outcome. Different from previous studies, PD1 positivity has no significant association with the $\mathrm{BCR}$ in high risk prostate cancer received AHT after RP with the hazard ratio 0.317 (95\% CI: 0.186-1.134; $\mathrm{p}=0.094)$ in localized disease and 2.746 (95\% CI: 0.733-10.33; $\mathrm{p}=0.135$ ) in metastatic disease. We combined PDL1/PD1 status as a panel to further analyze its prognosis value and strikingly concluded that the hazard ratio for BCR progression with the panel of PDL1/PD1 status expression in localized disease was 6.330 (95\% CI: 2.843-14.095; $\mathrm{p}<0.001)$.

The main limitation of our study was its retrospective and observational nature, including its single-institution with limited amount of cases. The median follow-up time from diagnosis to data analysis was 40 months (IQR: 29-53 months), which is limited to observe the endpoint (44 of 127 patients progressed to BCR) and 2 patients died of prostate cancer. The restrictive amount of endpoint events could raise serious concerns about the statistical power and utility of the multivariate analysis. More prospective, multicenter, large-scale trials are warranted to verify these results. In addition, our patients were recruited in a long period, during which the adjuvant hormonal therapy (48 received maximal androgen blockade, 37 received castration and 42 received anti-androgen therapy) and the radical prostatectomy (52 received laparoscopic RP, 39 received robot-assistant $\mathrm{RP}$ and 36 received traditional RP) were various. As a semi-quantitative method, the sensitivity and specificity of IHC is the main concern. This monoclonal PDL1 antibody we applied showed diffuse nuclear staining uniformly present in both benign stroma and neoplastic cells which is contrast to the membranous staining pattern reported by Haffner et $\mathrm{al}^{31}$. We validated its specificity by siRNA and western-blotting. IHC was applied in positive and negative FFPE tissue controls. A commonly stain intensity score was evaluated and the cut-off value was determined from previous studies ${ }^{11}$, 19. To avoid the bias and compression, the raw data of 
stain intensity score was presented in the Supplement. Several studies have indicated the correlation of AR and PDL1 expression both in CRPC and hormonal sensitive prostate cancer ${ }^{10}, 11$. Early evidence was found in Enzalutamide-resistant CRPC, PD1/PDL1 showed simultaneously high immune scores $^{35}$. However, the molecular mechanism between the androgen deprivation therapy and PD1/PDL1 pathway targeted therapy is still unknown. The blockade of PD1/PDL1 pathway might potentially be a novel alternative adjuvant treatment option for high-risk prostate cancer after radical prostatectomy.

Further studies are still needed to elucidate this assumption: what is the internal correlation between ADT and PD1/PDL1 pathway; how to optimize the PD1/PDL1 assay in high-risk prostate cancer and make it a novel biomarker to identify prostate cancer received immediate definitive therapy; what is the effect of PD1/PDL1 targeted therapy in prostate cancer and the predictive value of PDL1 expression for the response and prognosis to anti-PDL1 therapy, especially in high-risk prostate cancer.

\section{Conclusion}

Our study firstly demonstrated that PDL1 expression is not only highly prevalent in high-risk prostate cancer, but is also an independent factor in the prognosis of high-risk prostate cancer received AHT after RP. Strikingly, the combination of PDL1 and PD1 as a panel shows a significant prognosis value of BCR. These early clinical evidences could contribute to a more comprehensive understanding of the immune microenvironment of prostate cancer. In line with previous studies, our results indicated an association of PDL1 with adverse prognosis in high-risk primary prostate cancer, proposed PD1/PDL1 pathway targeted therapy a potentially adjuvant treatment option for high-risk prostate cancer after radical prostatectomy. Immunohistochemical detection of PD1/PDL1 could be a biomarker for the distinction of patients applicable for this therapy.

\section{Abbreviations}

PD1: programmed death 1; PDL1: programmed death ligand 1; AHT: adjuvant hormonal therapy; RP: radical prostatectomy; PSA: prostate specific antigen; TTN: time to PSA nadir; BCR: biochemical recurrence; HR: Hazard ratio; CI: Confidence interval; CRPC: castration-resistant prostate cancer; CT: computed tomography.

\section{Supplementary Material}

Supplementary methods, figures and table. http://www.jcancer.org/v10p3102s1.pdf

\section{Acknowledgments}

We thank all investigators and staff at the participating institutions, team members of the pathology department of Tongji Hospital and, above all, the patients, without whom this work would not have been possible. This work was supported by grants from the National Natural Science Foundation of China (81470935, 81370805, 81670645, 81602236), the Chenguang Program of Wuhan Science and Technology Bereau (2015070404010199, 2015071704 021644).

\section{Author contributions}

Z.W. and H.L. acquired, analyzed and interpreted the data and drafted the manuscript, Z.Y. and H.X.designed and supervised the study. Y.Z. and B.D. reviewed Haematoxylin and eosin sections to determine the presence of representative areas of the original samples and to confirm tumor histology and grade. H.L., L.Y. and W.G. prepared all figures, Z.H., X.Z., G.S. and S.W. edited all tables. All authors reviewed and approved the manuscript.

\section{Data availability statement}

The datasets generated during and/or analysed during the current study are available from the corresponding author on reasonable request.

\section{Competing Interests}

The authors have declared that no competing interest exists.

\section{References}

1. Ferlay J, Soerjomataram I, Dikshit R, et al. Cancer incidence and mortality worldwide: sources, methods and major patterns in GLOBOCAN 2012. International journal of cancer Journal international du cancer. 2015; 136: E359-386.

2. Siegel R, DeSantis C, Virgo $K$, et al. Cancer treatment and survivorship statistics, 2012. CA: a cancer journal for clinicians. 2012; 62: 220-241.

3. Heidenreich A, Bastian PJ, Bellmunt J, et al. EAU guidelines on prostate cancer. part 1: screening, diagnosis, and local treatment with curative intent-update 2013. European urology. 2014; 65: 124-137.

4. See WA, Wirth MP, McLeod DG, et al. Bicalutamide as immediate therapy either alone or as adjuvant to standard care of patients with localized or locally advanced prostate cancer: first analysis of the early prostate cancer program. The Journal of urology. 2002; 168: 429-435.

5. Messing EM, Manola J, Sarosdy M, et al. Immediate hormonal therapy compared with observation after radical prostatectomy and pelvic lymphadenectomy in men with node-positive prostate cancer. The New England journal of medicine. 1999; 341: 1781-1788.

6. Messing EM, Manola J, Yao J, et al. Immediate versus deferred androgen deprivation treatment in patients with node-positive prostate cancer after radical prostatectomy and pelvic lymphadenectomy. The Lancet Oncology. 2006; 7: 472-479.

7. Carosella ED, Ploussard G, LeMaoult J, et al. A Systematic Review of Immunotherapy in Urologic Cancer: Evolving Roles for Targeting of CTLA-4, PD-1/PD-L1, and HLA-G. European urology. 2015; 68: 267-279.

8. Okazaki T, Chikuma S, Iwai Y, et al. A rheostat for immune responses: the unique properties of PD-1 and their advantages for clinical application. Nature Immunology. 2013; 14: 1212

9. Dong H, Strome SE, Salomao DR, et al. Tumor-associated B7-H1 promotes T-cell apoptosis: A potential mechanism of immune evasion. Nature medicine. 2002; 8: 793.

10. Topalian SL, Hodi FS, Brahmer JR, et al Safety, activity, and immune correlates of anti-PD-1 antibody in cancer. The New England journal of medicine. 2012; 366: 2443-2454

11. Gevensleben H, Dietrich D, Golletz C, et al. The Immune Checkpoint Regulator PD-L1 Is Highly Expressed in Aggressive Primary Prostate Cancer. 
Clinical cancer research : an official journal of the American Association for Cancer Research. 2016; 22: 1969-1977.

12. Ness N, Andersen S, Khanehkenari MR, et al. The prognostic role of immune checkpoint markers programmed cell death protein 1 (PD-1) and programmed death ligand 1 (PD-L1) in a large, multicenter prostate cancer cohort. Oncotarget. 2017; 8: 26789-26801.

13. Goltz D, Gevensleben $\mathrm{H}$, Dietrich J, et al Promoter methylation of the immune checkpoint receptor PD-1 (PDCD1) is an independent prognostic biomarker for biochemical recurrence-free survival in prostate cancer patients following radical prostatectomy. Oncoimmunology. 2016; 5: e1221555.

14. Chen X, Bernemann C, Tolkach Y, et al. Overexpression of nuclear AR-V7 protein in primary prostate cancer is an independent negative prognostic marker in men with high-risk disease receiving adjuvant therapy. Urologic oncology. 2017; 36: 161.e19-161.e30.

15. Cornford P, Bellmunt J, Bolla M, et al. EAU-ESTRO-SIOG Guidelines on Prostate Cancer. Part II: Treatment of Relapsing, Metastatic, and Castration-Resistant Prostate Cancer. European urology. 2017; 71: 630-642.

16. Chang K, Qin XI, Zhang HL, et al. Comparison of two adjuvant hormone therapy regimens in patients with high-risk localized prostate cancer after radical prostatectomy: primary results of study CU1005. Asian journal of andrology. 2016; 18: 452-455.

17. Scher HI, Morris MJ, Stadler WM, et al. Trial Design and Objectives for Castration-Resistant Prostate Cancer: Updated Recommendations From the Prostate Cancer Clinical Trials Working Group 3. Journal of clinical oncology : official journal of the American Society of Clinical Oncology. 2016; 34: 1402-1418.

18. Geethakumari PR, Cookson MS, Kelly WK. The Evolving Biology of Castration-Resistant Prostate Cancer: Review of Recommendations From the Prostate Cancer Clinical Trials Working Group 3. Oncology (Williston Park, NY). 2016; 30: 187-195, 199.

19. D'Incecco A, Andreozzi M, Ludovini V, et al. PD-1 and PD-L1 expression in molecularly selected non-small-cell lung cancer patients. British journal of cancer. 2015; 112: 95-102.

20. Huggins C, Hodges CV. Studies on prostatic cancer. I. The effect of castration, of estrogen and androgen injection on serum phosphatases in metastatic carcinoma of the prostate. CA: a cancer journal for clinicians. 1972; 22: 232-240.

21. Engel J, Bastian PJ, Baur H, et al. Survival benefit of radical prostatectomy in lymph node-positive patients with prostate cancer. European urology. 2010; 57: 754-761.

22. Ghavamian R, Bergstralh EJ, Blute ML, et al. Radical retropubic prostatectomy plus orchiectomy versus orchiectomy alone for pTxN+ prostate cancer: a matched comparison. The Journal of urology. 1999; 161: 1223-1227; discussion 1227-1228.

23. McLeod DG, Iversen $P$, See WA, et al. Bicalutamide $150 \mathrm{mg}$ plus standard care vs standard care alone for early prostate cancer. BJU international. 2006; 97: 247-254.

24. Wong YN, Freedland S, Egleston B, et al. Role of androgen deprivation therapy for node-positive prostate cancer. Journal of clinical oncology : official journal of the American Society of Clinical Oncology. 2009; 27: 100-105.

25. Horwich A, Parker C, de Reijke T, et al. Prostate cancer: ESMO Clinical Practice Guidelines for diagnosis, treatment and follow-up. Annals of oncology : official journal of the European Society for Medical Oncology / ESMO. 2013; 24 Suppl 6: vi106-114.

26. Li H, Wang Z, Tang K, et al. Prognostic Value of Androgen Receptor Splice Variant 7 in the Treatment of Castration-resistant Prostate Cancer with Next generation Androgen Receptor Signal Inhibition: A Systematic Review and Meta-analysis. European urology focus. 2017; 4: 529-539.

27. Callahan MK, Postow MA, Wolchok JD. Targeting T Cell Co-receptors for Cancer Therapy. Immunity. 2016; 44: 1069-1078.

28. Sundar R, Cho BC, Brahmer JR, et al. Nivolumab in NSCLC: latest evidence and clinical potential. Ther Adv Med Oncol. 2015; 7: 85-96.

29. Mahoney KM, Rennert PD, Freeman GJ. Combination cancer immunotherapy and new immunomodulatory targets. Nat Rev Drug Discov. 2015; 14: 561-584.

30. Martin AM, Nirschl TR, Nirschl CJ, et al. Paucity of PD-L1 expression in prostate cancer: innate and adaptive immune resistance. Prostate cancer and prostatic diseases. $2015 ; 18: 325-332$.

31. Haffner MC, Guner G, Taheri D, et al. Comprehensive Evaluation of Programmed Death-Ligand 1 Expression in Primary and Metastatic Prostate Cancer. The American journal of pathology. 2018; 188: 1478-1485.

32. Fankhauser CD, Schuffler PJ, Gillessen $S$, et al. Comprehensive immunohistochemical analysis of PD-L1 shows scarce expression in castration-resistant prostate cancer. Oncotarget. 2018; 9: 10284-10293.

33. $\mathrm{Li} \mathrm{H}$, Wang $\mathrm{Z}$, Xiao $\mathrm{W}$, et al. Androgen-receptor splice variant-7-positive prostate cancer: a novel molecular subtype with markedly worse androgen-deprivation therapy outcomes in newly diagnosed patients. Modern pathology: an official journal of the United States and Canadian Academy of Pathology, Inc. 2018; 31: 198-208.

34. Gevensleben H, Holmes EE, Goltz D, et al. PD-L1 promoter methylation is a prognostic biomarker for biochemical recurrence-free survival in prostate cancer patients following radical prostatectomy. Oncotarget. 2016; 7:79943-79955.

35. Massari F, Ciccarese C, Calio A, et al. Magnitude of PD-1, PD-L1 and T Lymphocyte Expression on Tissue from Castration-Resistant Prostate Adenocarcinoma: An Exploratory Analysis. Targeted oncology. 2016; 11: 345-351. 